\title{
Light Illumination Induces Large Change in the Higher Order Structure of Giant DNAs Complexed with Maltosylated Amino-Pendant Polyethylene Glycol and Sodium Dodecyl Sulfate
}

\author{
Yuko YoshIKAWA, Yoshiyuki KoYAMA*, and Kenichi YosHIKAWA**, \\ Department of Food and Nutrition, Nagoya Bunri College, Nagoya 451-0077, Japan \\ * Department of Home Economics, Otsuma Women's University, Tokyo 102-8357, Japan \\ ${ }^{* *}$ Department of Physics, Graduate School of Science, Kyoto University, Kyoto 606-8502, Japan
}

(Received February 22, 1999)

\begin{abstract}
We have performed single-chain observation by fluorescence microscopy on T4 DNAs complexed with maltosylated amino-pendant polyethylene glycol (mal-PEG-A) in the presence of sodium dodecyl sulfate (SDS). With $0.02 \mathrm{mM}$ mal-PEG-A/SDS $([\mathrm{NH}]=[\mathrm{SDS}]=0.02 \mathrm{mM})$, individual DNAs existed as either a compact globule state or a partially unfolded globule state. Aggregates between multiple number of DNA chains are formed at higher concentrations of mal-PEG-A/SDS. With $1 \mathrm{mM}$ mal-PEG-A/SDS, the DNAs take an elongated coil conformation. It was found that the aggregate with multiple DNAs changes its morphology markedly during the focused illumination of $400-500 \mathrm{~nm}$ light. Such a morphological change of the complex has been discussed in relation to the ability of SDS micelle to anchor the DNA fragments induced by the cutting with photo-illumination .

KEY WORDS Higher Order Structure of DNA / Coil-Globule Transition / Single Molecular Observation / Fluorescence Microscopy / Light Induced Conformational Change / Giant DNA / maltosylated PEG/
\end{abstract}

In living cells, giant DNAs exist in highly compacted states. $^{1-5}$ Whereas, isolated DNAs take elongated coiled states in aqueous solutions, because water is a good solvent for negatively charged DNA chains. It is well known that DNAs tend to aggregate and precipitate with the addition of various condensing agents such as polyamines and neutral polymers. ${ }^{6-15}$ Generally, the term condensation has been used for situations where a finite number of DNA molecules are compacted. It has been rather difficult to study the compaction of single DNA in its genuine meaning due to the limitation of the available experimental techniques such as light scattering, sedimentation and CD spectrometry.

Recently, from the direct observation of individual duplex DNA molecules using fluorescence microscopy, ${ }^{16}$ we found that isolated giant DNA chains undergo a transition between an elongated coiled state and a collapsed compact state. ${ }^{17-19}$ The essential aspects of the transition of single DNA chains are summarized as follows: (1) Individual DNA chains, more than several tens of kilo base pairs long, exhibit a discrete transition between an elongated coil and a compact globule. The change in the packing density of the segments in a DNA chain is more than an order of $10^{4}$. This transition is reversible. (2) The discrete nature of the coil-globule transition is rather general, and the transition can be induced by various kinds of condensation agents such as neutral hydrophilic polymer (PEG), cationic surfactant (CTAB), alcohol, multivalent inorganic cation, and polyamine (spermidine and spermine), etc. (3) The diagram of the transition with respect to the condensation shows a region where the coil and globule state coexist.

More recently, we have performed the study on the change of the higher order structure of giant DNAs induced by a polyethylene glycol derivative with pendant amino

\footnotetext{
${ }^{\dagger}$ To whom correspondence should be addressed.
}

groups (PEG-A). ${ }^{20}$ It has been found that segregation between collapsed and elongated regions in a duplex DNA chain is generated as an intermediate state in the collapsing process induced by PEG-A. This is in contrast to the allor-none transition by PEG. In the present study, we have examined the effect of maltosylated PEG-A (mal-PEG-A) on the higher order structure of DNAs as an extension of our last study on the PEG-A complex of DNA. We have prepared mal-PEG-A with the expectation that the maltosyl moiety would create novel biological activity. Because in biological sy stems, sugar residues are normally conjugated to proteins or lipids and serve as recognition structures and integral components of cell growth-regulating networks. ${ }^{21}$ We have found that, with addition of sodium dodecyl sulfate (SDS), mal-PEG-A form stable micelle structures in aqueous solution. Thus, in the present study, we have investigated the aggregation behavior of the complex of DNA with malPEG-A in the presence of SDS.

\section{EXPERIMENTAL}

\section{Materials}

Maltosy lated amino pendant polyethylene glycol $(\mathrm{Mn}=$ 3700, 4.7 amino groups/polymer molecule, Figure 1) was synthesized from amino pendant poly ethylene gly col (PEGA) and maltose in the presence of $\mathrm{NaBH}_{3} \mathrm{CN}$, and was purified with dialysis. The sample obtained was then mixed with sodium dodecyl sulfate (SDS) in water at a molar ratio of $\mathrm{NH} / \mathrm{SO}_{4}=1 . \quad \mathrm{T} 4$ phage DNA was purchased from Nippon Gene (Toyama, Japan). The fluorescent dye 4',6diamidino-2-phenylindole (DAPI) and the antioxidant 2mercaptoethanol (2-ME) were purchased from Wako Pure Chemical Industries (Osaka, Japan).

\section{Fluorescence microscopic measurements}

T4 DNA, $166 \mathrm{kbp}$ with a contour length of $57 \mu \mathrm{m}$, was dissolved in pure water, and DAPI and 2-ME were added to 
the DNA solution. The final concentrations were as follows: $3 \mu \mathrm{M}$ T4 DNA (in nucleotide units), $1 \mu \mathrm{M}$ DAPI, and $4 \%(v / v) 2-M E$. It has been confirmed that the persistence length and the contour length of DNA remain essentially constant at such a low concentration of DAPI. Compaction was induced by the addition of mal-PEG-A/SDS to the DNA solution. Fluorescence DNA images were obtained using an Axiovert 135 TV microscope (Carl Zeiss, Germany) equipped with a $100 \mathrm{x}$ oil-immersion objective lens and a high-sensitivity Hamamatsu SIT TV camera, that allowed recording of images on video tapes. The video image was analyzed with an Argus 50 image processor (Hamamatsu Photonics, Hamamatsu, Japan). Observation was performed at $20^{\circ} \mathrm{C}$.

\section{RESULTS AND DISCUSSION}

We have performed fluorescence microscopic observations on T4 DNA molecules with various concentrations of the mal-PEG-A/SDS, where $[\mathrm{NH}]=[\mathrm{SDS}]$ have been changed from $0.01 \mathrm{mM}$ to $1 \mathrm{mM}$. Without mal-PEG-A/SDS, individual DNA molecules were observed as random coil in aqueous solution (Figure $2 \mathrm{a}$ ). In the presence of $0.02 \mathrm{mM}$ mal-PEG-A/SDS, individual DNA molecules existed as either a globule state or a partial globule state. In the partial globule state, a flexible coiled part was connected to a compacted globule part (Figure $2 b$ ). Such kind of collapsing process is similar to that induced by PEG-A. ${ }^{20}$ In contrast, the transition induced by PEG is all-or-none; individual DNA molecules exhibit either elongated coil or completely collapsed globule. ${ }^{17}$ With increasing concentration of malPEG-A/SDS, aggregates between multiple DNA chains are formed. Figure $2 \mathrm{c}$ shows a multi-chain aggregate in the presence of $0.1 \mathrm{mM}$ mal-PEG-A/SDS. These aggregates are dissolved in the aqueous phase by avoiding the adsorption onto the slide glass surface. . It is found that mal-PEGA/SDS yields a critical micelle concentration $(\mathrm{cmc})$ value in the range of $0.1-0.3 \mathrm{mM}$. Under the condition of $1 \mathrm{mM}$ mal-PEG-A/SDS, DNA molecules change their conformation to elongated coil again. Thus, it has become clear that with mal-PEG-A/SDS, DNA chains change from elongated coil into partial globule (intrachain segregated state) and into compact globule, as is schematically represented in Figure 5. Then, the complex tends to form multi-chain aggregate.

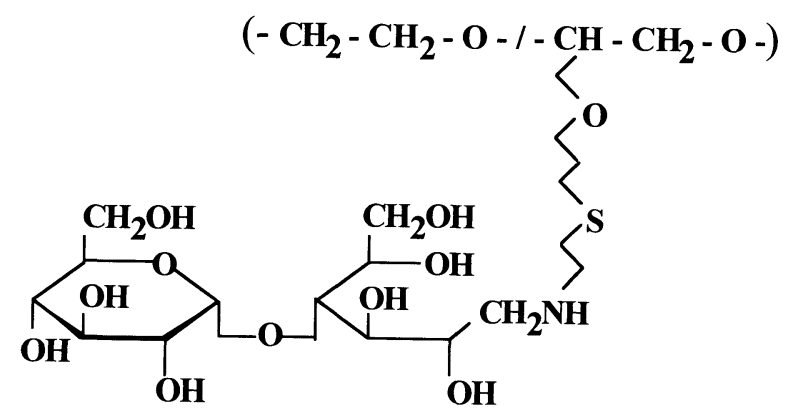

Figure 1. Structure of maltosylated amino pendant polyethylene glycol (mal-PEG-A).
Finally, at the concentration of mal-PEG-A/SDS much higher than the cmc, the complex changes its conformation into random coil again.

Interestingly, the multi-chain aggregate changes its morphology markedly during the illumination of $400-500 \mathrm{~nm}$ light as is shown in Figures 3 and 4 . It has been confirmed that such morphological change is irreversible, in other words, the light-induced change in the apparent morphology does not go back to the initial morphology ever after the switching off of the illumination.

The fluorescence microscopic observation of DNA chains with mal-PEG-A in the absence of SDS has indicated that, although similar morphology in the DNA multi-chain aggregate is observed, the complex gradually degrades into small fragments under illumination of $400-500 \mathrm{~nm}$ light. Such

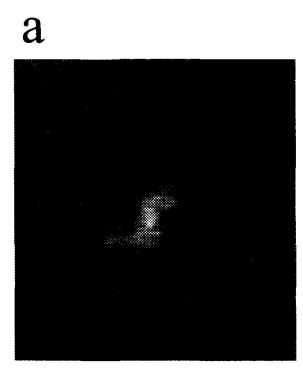

b
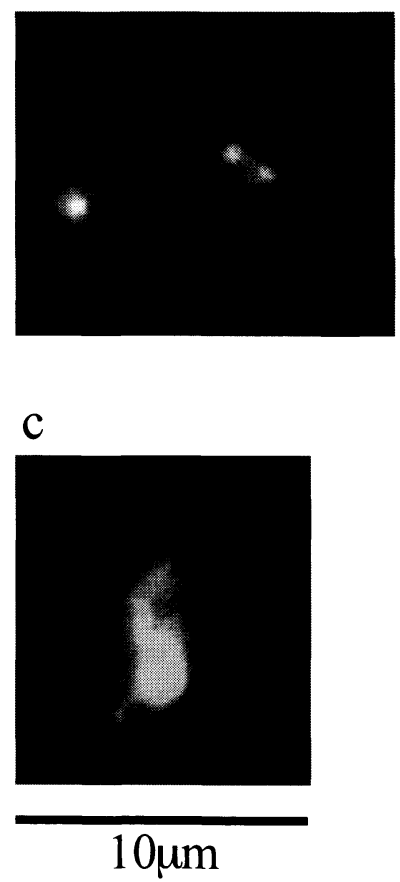

Figure 2. Fluorescence microscopic images of T4 DNA complexed with mal-PEG-A/SDS moving freely in aqueous solution. a; Without mal-PEG-A/SDS, DNAs exibit elongated coil, b; with $0.02 \mathrm{mM}$ malPEG-A/SDS, DNAs take compact globule (left) and partial globule (right), c; with $0.1 \mathrm{mM}$ mal-PEG-A/SDS, multi-chain aggregate is formed. The concentrations are given by those of amino group.

Polym. J., Vol. 31, No. 11-2, 1999 
$\mathrm{a}$
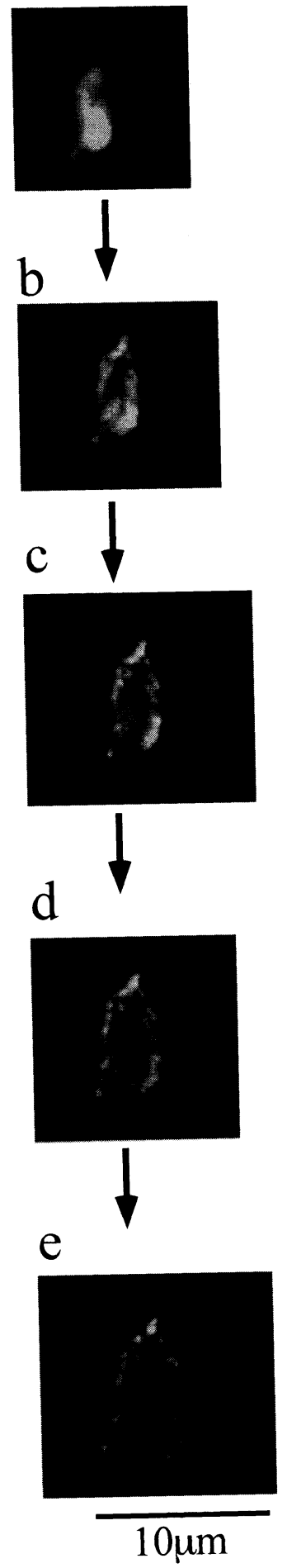

Figure 3. Time-course of morphological change in the multi-chain aggregate induced by photo-illumination, as observed by fluorescence microscopy (a-e). The time interval between the pictures is ca. $1 \mathrm{~s}$.

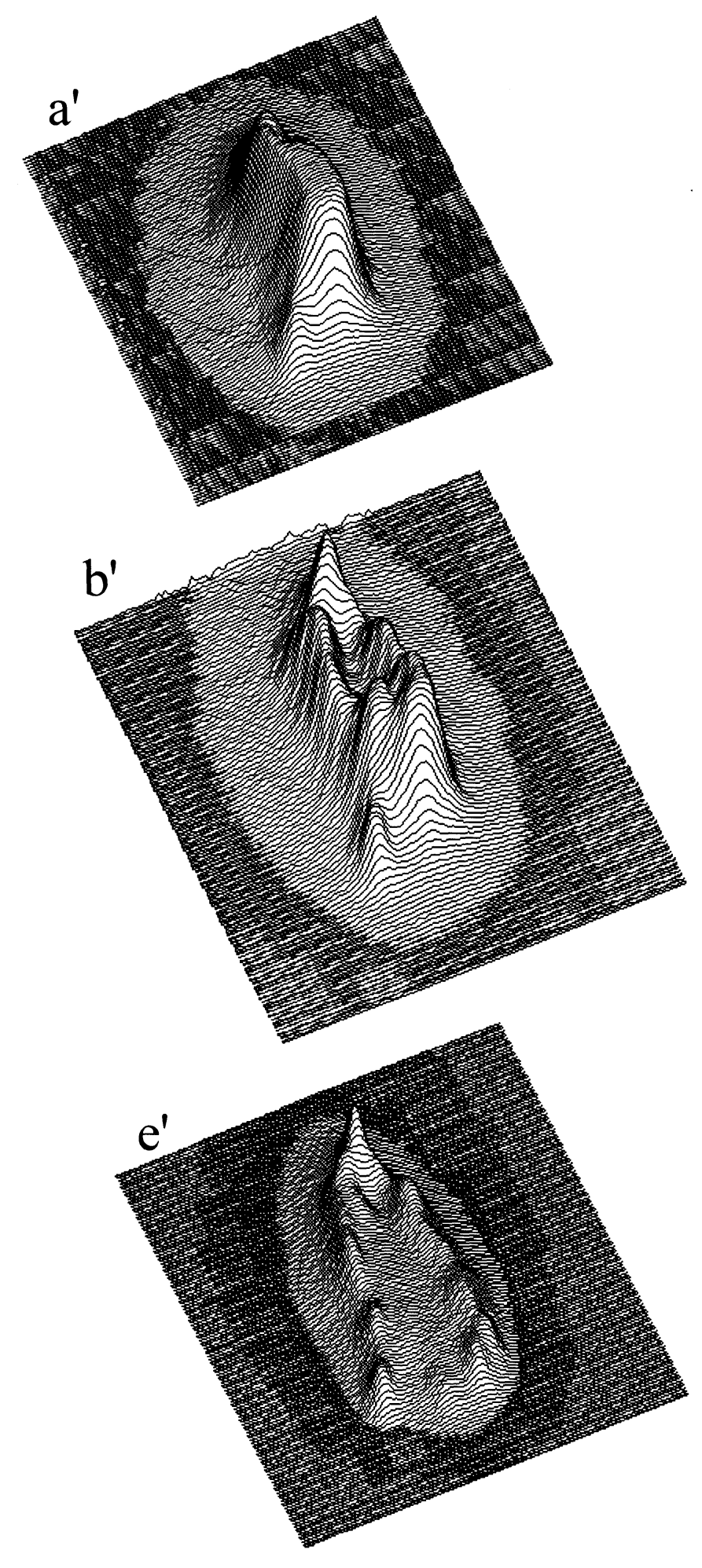

Figu re 4. Light-intensity distribution in the multi-chain aggregate: $a^{\prime}, b^{\prime}$ and $\mathrm{e}^{\prime}$ correspond to the pictures of $\mathrm{a}, \mathrm{b}$ and $\mathrm{e}$ in Figure 3, respectively. 


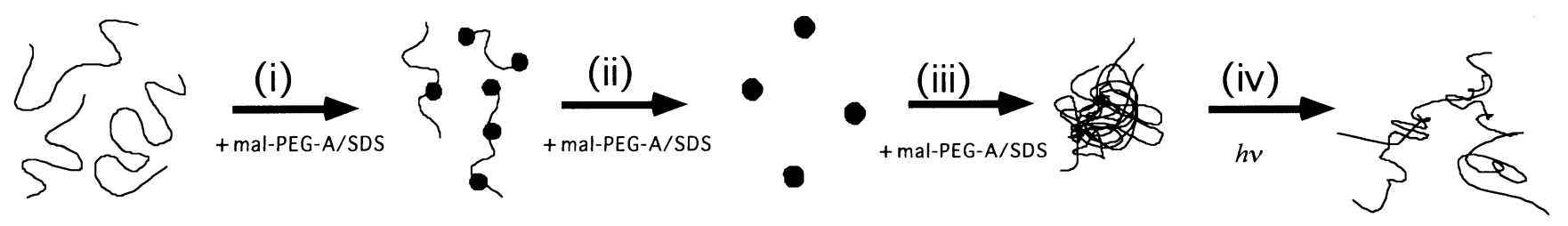

Figure 5. Schematic representation of the morphological change of DNAs. With the increase of concentration of mal-PEG-A/SDS, (i) the DNAs change the conformation from elongated coil into partial globule, i.e., intrachain segregated state, then (ii) the partial globule changes into completely packed globule, and (iii) the globular DNAs become to form an assembly. (iv) With photo-illumination, the assembly changes the structure to be an open form.

fragmentation of the complex is attributable to the effect of the focused light of the illumination to cut the double stranded DNA chains. Thus, the assembling effect of SDS may anchor the fragments of DNA chains in the presence of mal-PEG-A/SDS. As a result, the characteristic large morphological change would be generated, accompanied with the gradual decomposition of the long DNA chains induced by the illumination. To summarize the experimental results in the present study, it has become clear that characteristic morphological changes are generated on the DNA complex with photo-irradiation as is given schematically in Figure 5. With increase of the concentration of mal-PEG-A/SDS, the elongated coiled DNA chains change the higher order structure into fully collapsed compact state through the formation of the partial globule conformation. With the further increase of mal-PEG-A/SDS, aggregates of multiple DNA molecules are generated. The aggregated complex exhibits marked change in the morphology with photoillumination. As a future extension, it may be interesting to study the effect of the photo-illumination on the degree of degradation of DNA molecules.

Acknowledgment. The present study is supported by Grant-in-Aid for Scientific Research on Priority Areas, "New Polymers and Their Nano-Organized Systems," from the Ministry of Education, Science, Sports and Culture of Japan.

\section{REFERENCES}

1. V. A. Bloomfield, Biopolymers, 31, 1471 (1991).

2. R. Marquet and C. Houssier, J. Biomol. Struct. Dyn., 9, 159(1991).

3. L. D. Murphy and S. B. Zimmerman, Biophys. Chem., 57, 71 (1995).

4. J. Pelta, D. Durand, J. Doucet, and F. Livolant, Biophys. J., 71, 48 (1996).

5. V. A. Bloomfield, Curr. Opin. Struct. Biol., 6, 334 (1996).

6. L. S. Lerman, Proc. Natl. Acad. Sci. USA, 68, 1886 (1971).

7. U. K. Laemmli, Proc. Natl. Acad. Sci. USA, 72, 4288 (1975).

8. L. C. Gosule and J. A. Shellmann, Nature, 259, 333 (1976).

9. D. K. Cattoraji, L. C. Gosule, and J. A. Shellmann, J. Mol. Biol., 12, 327 (1978).

10. J. Widom and R. L. Baldwin, J. Mol. Biol., 144, 431 (1980).

11. C. Reich, M. F. Maestre, S. Edmomson, and D. M. Gray, Biochemistry, 19 , 5208 (1980).

12. R. Huey and S. C. Mohr, Biopolymers, 20, 2533 (1981).

13. J. Widom and R. L. Baldwin, Biopolymers, 22, 1595 (1983).

14. D. Porschke, Biochemistry, 23, 4821 (1984).

15. P. G. Arscott, C. Ma, J. R. Wenner, and V. A. Bloomfield, Biopolymers, 36, 345 (1995).

16. M. Yanagida, Y. Hiraoka, and I. Katsura, Cold Spring Harbor Symp. Quant. Biol., 47, 177 (1983).

17. K. Minagawa, Y. Matsuzawa, K. Yoshikawa, M. Matsumoto, and M. Doi, FEBS Lett., 295, 65 (1991).

18. S. M. Melnikov, V. G. Sergeyev, and K. Yoshikawa, J. Am.Chem. Soc., 117, 2401 (1995).

19. Y: Yoshikawa and K. Yoshikawa, FEBS Lett., 361, 277 (1995).

20. K. Yoshikawa, Y. Yoshikawa, Y. Koyama, and T. Kanbe, J. Am. Chem. Soc., 119, 6473 (1997).

21. T. Feizi, Biochem. Soc. Trans., 16, 930 (1988). 